\title{
BMJ Open Burden of diabetic macular oedema in patients receiving antivascular endothelial growth factor therapy in South Korea: a healthcare resource use and cost analysis
}

\author{
Ha-Lim Jeon (D) , ${ }^{1}$ Hyesung Lee (D) , ' Dongwon Yoon (D) , ${ }^{1}$ Yeonkyung Lee, ${ }^{2}$ \\ Jae Hui Kim (D) , ${ }^{3}$ Donghyun Jee, ${ }^{4}$ Ju-Young Shin (D) ${ }^{1}$
}

To cite: Jeon H-L, Lee $\mathrm{H}$, Yoon D, et al. Burden of diabetic macular oedema in patients receiving antivascular endothelial growth factor therapy in South Korea: a healthcare resource use and cost analysis. BMJ Open 2020;10:e042484. doi:10.1136/ bmjopen-2020-042484

- Prepublication history and additional material for this paper are available online. To view these files, please visit the journal online (http://dx.doi. org/10.1136/bmjopen-2020042484).

Received 06 July 2020 Revised 09 November 2020 Accepted 04 December 2020

\section{Check for updates}

(C) Author(s) (or their employer(s)) 2020. Re-use permitted under CC BY-NC. No commercial re-use. See rights and permissions. Published by BMJ.

${ }^{1}$ School of Pharmacy, Sungkyunkwan University, Suwon, South Korea ${ }^{2}$ Bayer Korea Ltd, Seoul, South Korea

${ }^{3}$ Department of Ophthalmology, Kim's Eye Hospital, Konyang University College of Medicine, Seoul, South Korea

${ }^{4}$ Department of Ophthalmology, St. Vincent's Hospital, College of Medicine, Catholic University of Korea, Seoul, South Korea

Correspondence to Professor Ju-Young Shin; shin.jy@skku.edu

\section{ABSTRACT}

Objective To examine healthcare resource utilisation $(\mathrm{HRU})$ and direct medical costs for patients with diabetic macular oedema (DME) treated with antivascular endothelial growth factor (anti-VEGF) in Korea by comparing with those for (1) patients with diabetes mellitus (DM) without retinopathy and (2) patients with neovascular age-related macular degeneration (nAMD) treated with anti-VEGF.

Design Retrospective cohort study.

Setting The Korean National Health Insurance (NHI) database from 1 January 2014 to 31 December 2016. Participants We identified 1398 patients older than 30 years of age who received anti-VEGF treatment for DME in 2015 after excluding patients who had a diagnosis of nAMD in 2015 and any cancer in the preceding year.

Main outcome measures One-year healthcare resource use and direct medical costs of patients with DME treated with anti-VEGF.

Results In total, 1398 patients with DME receiving antiVEGF, 12813 patients with DM without retinopathy and 12 222 patients with nAMD receiving anti-VEGF were identified. Hospital admissions and outpatient visits were highest in patients with DME, while the number of licensed anti-VEGF injections in those with DME was about half that of those with nAMD (2.1 vs 3.9 per patient per year). Mean 1-year medical costs were also higher in patients with DME (US\$6723) than in those with DM without retinopathy (US\$2687) and nAMD (US\$4980). In a multivariable analysis with matched cohorts, DME was associated with $66 \%$ higher medical costs for comorbid diseases (adjusted OR (aOR), 1.66; 95\% Cl 1.45 to $1.90)$ and $50 \%$ lower anti-VEGF injections (aOR, $0.50 ; 95 \% \mathrm{Cl}$ 0.46 to 0.54 ) compared with nAMD.

Conclusions The overall HRU and economic burden for DME treated with anti-VEGF were higher than for DM without retinopathy or for nAMD treated with anti-VEGF. Meanwhile, the lower number of licensed anti-VEGF injections compared with nAMD may reflect a potential lack of ophthalmological treatment for DME supported by the NHI in Korea.

\section{INTRODUCTION}

Diabetic macular oedema (DME) is an ocular complication of diabetes mellitus (DM) that may result in vision loss or blindness and
Strengths and limitations of this study

- This is the first study to examine healthcare resource utilisation and direct medical costs of patients with diabetic macular oedema (DME) treated with antivascular endothelial growth factor (anti-VEGF) in Korea by comparing them with two disease groups (patients with diabetes mellitus without retinopathy and patients with neovascular age-related macular degeneration (nAMD) treated with anti-VEGF).

- To reduce biases in estimating the effect of DME, we used a generalised linear model after applying exact matching and propensity score matching.

- As the clinical characteristics of patients with DME treated with anti-VEGF and those with nAMD treated with anti-VEGF are quite different, residual confounding can still exist despite application of various statistical approaches.

- Further studies considering the use of bevacizumab, an anti-VEGF agent used off-label, should be conducted to grasp healthcare resource utilisation and direct medical costs in patients with DME or nAMD more accurately.

thus significantly decrease patients' quality of life. ${ }^{1-3}$ According to previous research, $13 \%-25 \%$ of patients with type 1 or $2 \mathrm{DM}$ developed DME over a 10 -year period. ${ }^{4}$ Moreover, the 25-year cumulative incidence of DME was $29 \%$, with an incidence of approximately 75000 new patients annually in the USA. $^{5}{ }^{6}$ Given the growing prevalence of $\mathrm{DM},{ }^{78}$ the prevalence of DME is likewise expected to continue to increase.

A few studies have investigated the economic burden of DME. A cost-of-illness study using a cohort of US Medicare beneficiaries reported that $37.6 \%$ of patients with DME received laser photocoagulation 1.7 times over a 1-year period and that the 1-year direct medical cost was US\$11 290. ${ }^{2}$ In the study using US retrospective claims data based on a privately insured population, the 
annual total cost of DME was US $\$ 28606$, which was $75 \%$ higher than those of diabetic retinopathy without DME. ${ }^{9}$ A cross-sectional study in Germany showed that patients with DME visited ophthalmologists 7.3 times over 1 year and the total cost was $€ 1433 .{ }^{10}$ However, updated analyses are needed because these studies were conducted using data before the approval of ranibizumab and aflibercept and therefore do not reflect the use of these drugs.

Ranibizumab and aflibercept, which are licensed antivascular endothelial growth factor (anti-VEGF) drugs, were listed in the national drug formulary for treatment of DME by the Korean Ministry of Food and Drug Administration in 2015. Before the era of anti-VEGF antibody, macular laser treatment had been the gold standard of treatment for DME. ${ }^{11}$ Although the dexamethasone intravitreal implant has been reimbursed for DME by the National Health Insurance (NHI) since 2016, intravitreal injection of anti-VEGF is the most commonly used therapy for DME in Korea. ${ }^{13} 14$ Despite these dramatic changes, up-to-date information on healthcare resource utilisation (HRU) associated with and direct medical costs of DME is rare. Moreover, given that pro re nata (PRN) and treat-and-extend regimens are preferred for anti-VEGF treatment in routine practice over the fixed monthly or bimonthly injections used in clinical trials, ${ }^{15-17}$ it is necessary to investigate the yearly mean number of antiVEGF injections administered to patients with DME using real-world data.

Therefore, we aimed to estimate 1-year HRU and direct medical costs in total, including ophthalmology care and non-ophthalmology care, for patients with DME treated with anti-VEGF using nationwide claims data and compare them with those for patients with DM without retinopathy to better understand the HRU of patients with DME. In addition, we added one more comparison group, patients with neovascular age-related macular degeneration (nAMD) receiving anti-VEGF treatment. nAMD is the first approved indication of anti-VEGF treatment and, if untreated, may result in vision loss like DME. The clinical recommendations for using anti-VEGF in DME and nAMD are similar. According to the results from previous studies in other countries examining the number of anti-VEGF injections in patients with DME or nAMD using real-world data, the mean number of antiVEGF injections per year for DME was similar to those for nAMD. ${ }^{18-22}$ Generating data on HRU and medical costs of patients with DME in South Korea may be key to improve our understanding of the economic burden associated with DME and how anti-VEGFs are used to manage their disease. Also, comparison of total medical costs and HRU between patients with DME and nAMD could provide clinicians and policymakers meaningful insight into the economic burden as a whole in treating DME.

\section{METHODS}

\section{Data source}

We used 3-year data from the Korean NHI database (2014-2016). The NHI covers the entire South Korean population ( $97 \%$ by the NHI as compulsory social insurance and $3 \%$ by medical aid). The database includes demographic information such as sex, age and socioeconomic status of about 50 million inhabitants in Korea, as well as clinical information on prescribed drugs, procedures and disease diagnoses, which are coded according to the International Classification of Diseases, 10th revision (ICD-10), and treatment setting (inpatients or outpatients). Information on a prescribed drug includes the generic name, prescription date, duration of treatment and route of administration. It also includes information on the medical institution and medical costs incurred in hospitalisation or an outpatient visit. For administrative purposes, registration codes for the specific diseases are also recorded. The South Korean government implemented a policy to decrease the copayment for patients with rare and intractable diseases. A government agency, Health Insurance Review and Assessment Service, reviews patient eligibility and reliability of disease diagnosis; thus, using the registration code can be more accurate to select patients with the specific diseases than the ICD-10-based diagnosis code. ${ }^{23}$ A previous study showed the overall agreement between the diagnoses derived from the claims database and the actual diagnoses of inpatients' medical records from hospital or clinic to be $82.0 \%{ }^{24}$

\section{Study design and population}

We conducted a retrospective cohort study to generate 1-year HRU and cost estimates of DME. Patients older than 30 years of age who received anti-VEGF treatment for DME in 2015 were included in the study since anti-VEGFs have been approved for the treatment of DME in South Korea as of February 2015. Currently, a specific diagnosis code for DME does not exist in the South Korean healthcare system, and the diabetic retinopathy (H36.0) code has been used for reimbursement of medical service for DME. We defined patients treated with licensed antiVEGF, steroid or laser therapy with the diagnosis code for diabetic retinopathy as patients with advanced diabetic retinopathy including DME. We excluded patients who had a diagnosis of any cancer during the preceding year from the cohort because these patients may contribute to the overestimation of HRU and medical costs. After excluding patients who had nAMD in 2015, patients treated with anti-VEGFs were included in the final analysis. Patients were followed up for 1 year from the earliest date of anti-VEGF treatment for DME in 2015 (index date) (figure 1A). Eligible patients were required to have a diagnosis of DM (E10-E14) during the year before the index date.

Patients with DM without retinopathy were selected as the first comparison group. This group consisted of patients older than 30 years of age and who had a diagnosis code of DM (E10-E14) in 2015. Patients who had nAMD and any cancer during the preceding year were excluded. We selected patients using propensity score matching (1:10 matching) with patients with DME treated with anti-VEGF based on age and sex, due to the overly 
A
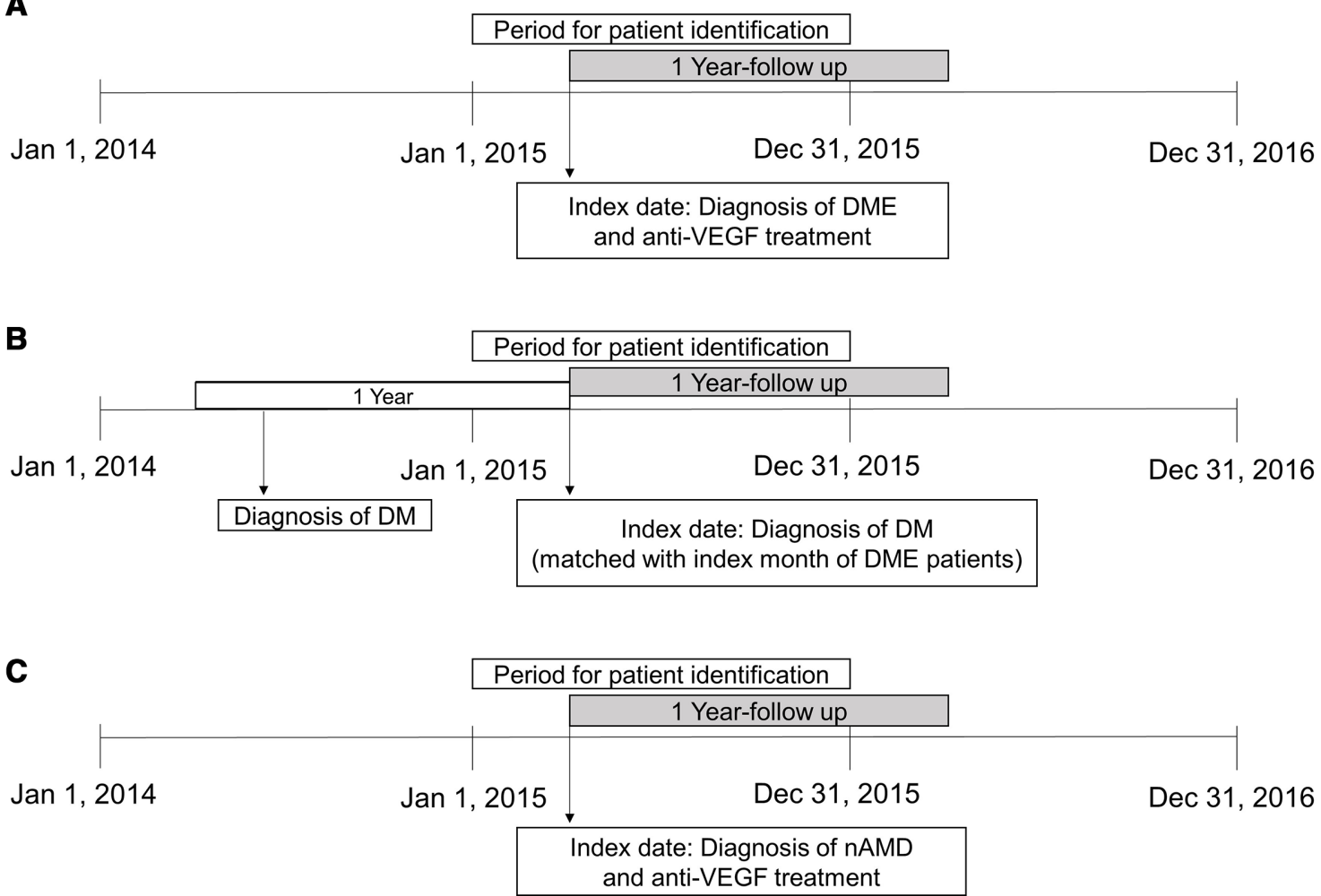

Figure 1 Study scheme and selection of patients with DME treated with anti-VEGF (A), DM without retinopathy (B), and nAMD treated with anti-VEGF (C). Anti-VEGF, antivascular endothelial growth factor; DM, diabetes mellitus; DME, diabetic macular oedema; nAMD, neovascular age-related macular degeneration.

large number of patients with DM without retinopathy in our database. We designated the index date as the date of DM record in 2015, of which the month was matched with that of the index date for patients with DME (figure 1B). Patients were eligible for inclusion in the study if they had no history of retinopathy $(\mathrm{H} 30-\mathrm{H} 36)$ during the year before the index date. Patients older than 30 years of age and who had nAMD treated with anti-VEGF were selected as the second comparison group. Because nAMD is covered by the copayment decreasing policy as an extrabenefit scheme for rare and intractable diseases, its registration code (V201) was used for patient selection. The index date for patients with nAMD was the earliest date when nAMD and anti-VEGF treatment were recorded at the same time in 2015 (figure 1C). Patients who had any cancer in 2015 and diabetic retinopathy during the preceding year were excluded.

\section{HRU and direct medical costs}

Regarding HRU, the total number of outpatient and ophthalmologist visits, the number of hospitalisations, the length of stay per hospitalisation and the number of anti-VEGF injections were computed during 1 year from the index date. We also calculated 1-year total medical costs using a gross-costing method, by summing all medical costs incurred in clinics or hospitals at the patient level. Since the extent of medical costs incurred in inpatients and outpatients can be different by disease groups, we examined the total costs in each setting. The costs in ophthalmology, the costs per outpatient/ophthalmologist visits and the costs for each disease of interest were also computed. The costs for each disease of interest indicate the medical costs for the treatment of DME in the DME group, the treatment of DM in the DM without retinopathy group and the treatment of nAMD in the nAMD group. We also calculated the medical costs for other diseases by subtracting the medical costs for each disease of interest from the 1-year total medical cost in the matched cohorts to examine the economic burden due to other diseases in each patient group. All costs were converted from Korean won to US dollars based on the average exchange rate in 2015 (US $\$ 1=1179.1$ Korean won). ${ }^{25}$

\section{Statistical analysis}

As the primary analysis, baseline characteristics were investigated to identify differences among the three groups. Age, sex, income-based insurance contribution levels (11 levels) as a proxy for socioeconomic status, residential district, and location and type of medical institution were assessed on the index date. Charlson Comorbidity Index (CCI) scores and comorbidity conditions (cataract, cerebrovascular disease, coronary heart disease, glaucoma, hypertension, peripheral vascular disease and renal disease) were identified for the year before the index date. In addition, duration of DM ( $<5$ years, $5-9$ years and $\geq 10$ years) was identified using the 10 -year medical record 
before the index date for the DME treated with anti-VEGF group and for the DM without retinopathy group.

In the secondary analysis, we compared 1-year HRU and medical costs of patients with DME treated with antiVEGF with those of patients with DM without retinopathy or of patients with nAMD using exact matching, propensity score matching and adjustment with a generalised linear model (GLM). Comparison of resource consumption and costs between different disease groups in observational studies is prone to bias due to the fundamental differences in the characteristics of each group. ${ }^{26}$ Therefore, a three-step approach was applied to minimise bias and to estimate the disease-specific costs attributable to DME. First, for the comparison between DME and DM without retinopathy groups, patients were exact-matched based on age, sex, duration of DM and the index month. In the comparison between DME and nAMD groups, patients were matched based on age, sex, CCI score and the index month. Second, subsequent matching with propensity score was applied to the exact-matched cohorts. A propensity score is the conditional probability of assignment to a particular treatment given a vector of observed covariates. ${ }^{27}$ In this study, we calculated the propensity score for the possibility of a diagnosis of DME through multiple logistic regression analysis using demographic and clinical baseline characteristics, such as age, sex, income level, CCI score and comorbidities. Variables related to HRU and costs for the year before the index date (1-year medical cost, number of outpatient visits, hospitalisations and mean length of stay per hospitalisation) were also included in the calculation of propensity score. A greedy matching method was used to match patients with DME treated with anti-VEGF with patients with DM without retinopathy or patients with nAMD treated with anti-VEGF. Standardised difference was calculated to examine the balance of covariate distribution between groups (absolute value greater than 0.1 considered as imbalance). Third, to estimate the effect of DME on HRU and medical costs relative to DM without retinopathy and nAMD treated with anti-VEGF, we calculated the OR for HRU and cost ratios with 95\% CI using a GLM with Poisson and gamma distribution, respectively. In these regression analyses, baseline characteristics including age, sex, income level, CCI score and all comorbidities were adjusted in model 1 , and variables related to HRU and costs for the year before the index date were added in model 2. These ratios refer to the relative increase or decrease in HRU and medical costs of patients with DME treated with anti-VEGF compared with patients with DM without retinopathy and patients with nAMD treated with anti-VEGF. All statistical analyses were performed in SAS V.9.4.

\section{Patient and public involvement}

Patients or the public were not involved in the design, conduct, reporting or dissemination plans of our research since we used de-identified participant data.

\section{RESULTS}

In total, 1398 patients with DME treated with anti-VEGF, 12813 patients with DM without retinopathy and 12 222 patients with nAMD treated with anti-VEGF were included in the final analysis. The mean (SD) age of patients with DME treated with anti-VEGF and patients with DM without retinopathy was 58 (10.9) years, and approximately $60 \%$ of the patients in these two groups were male (table 1 ). The mean (SD) age of patients with nAMD treated with anti-VEGF was 71.9 (8.9) years. The proportion of patients with a DM duration of $\geq 10$ years was considerably higher in the DME group than in the DM without retinopathy group (49.5\% vs $7.7 \%)$. In the DME and DM without retinopathy groups, $22.2 \%$ and $25.6 \%$ were recipients of medical aid, respectively, compared with $5.7 \%$ in the nAMD group. The mean (SD) CCI score in the DME group was $3.9(0.3)$, which was the highest CCI score among the groups. The overall proportion of patients with comorbidities was higher in the DME group than in the other groups.

One-year HRU and direct medical costs for each disease group are described in table 2 . The mean (SD) number of hospitalisations per patient among patients with DME with anti-VEGFs was 1.4 (2.9), and the mean (SD) length of stay per hospitalisation was 7.6 (7.5) days. The mean (SD) number of outpatient visits was 49.2 (35.8). The mean (SD) number of ophthalmologist visits was 9.1 (5.5) and that of anti-VEGF injections was 2.1 (1.5). The overall HRU of patients with DM without retinopathy and of patients with nAMD was lower than that of patients with DME, except in the case of the mean length of stay per hospitalisation among patients in the DM group (10.3; SD 9.0) and the mean number of anti-VEGF injections in the nAMD group (3.9; SD 2.0). In the subgroup analysis with patients with nAMD who did not receive intravitreal anti-VEGF injections during the year before the index date (anti-VEGF treatment-naive patients), the mean (SD) number of anti-VEGF injections was 4.1 (2.0) (data not shown).

The mean 1-year total medical cost (US\$6913; SD US\$8266) was higher in the DME group than in the other groups. The medical costs for the DM without retinopathy group were substantially lower than for the other groups. In the nAMD group, the mean (SD) ophthalmological care costs per patient and costs for each disease of interest were US $\$ 3777$ (US\$1814) and US $\$ 3720$ (US\$1877), which were higher than in the DME group.

After exact and propensity score matching of the DME and DM without retinopathy groups, 535 pairs of patients were generated. In the matching of patients with DME with patients with nAMD, 470 patients from each group were included. We observed that the distributions of covariates were balanced in the matched cohort (online supplemental tables S1 and S2). The HRU and direct medical costs of the matched sample of patients with DME, patients with DM without retinopathy and patients with nAMD are described in online supplemental tables S3-S6. 
Table 1 Sociodemographic and clinical characteristics of patients with DME treated with anti-VEGF, patients with DM without retinopathy and patients with nAMD treated with anti-VEGF

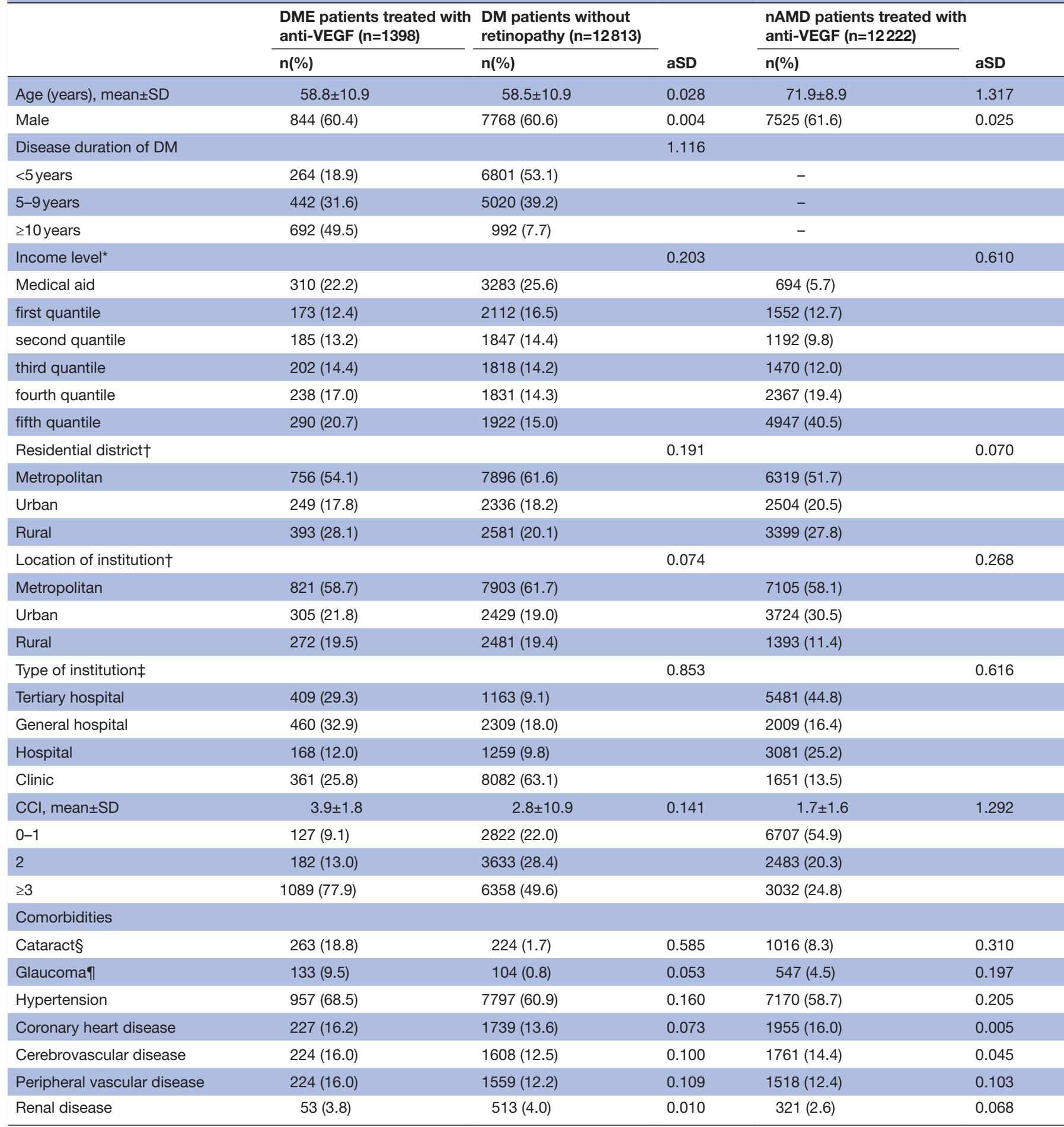

Any patient could be included as a duplicate patient in $>1$ group of treatment history for DME.

*Income levels were classified into six groups according to type of health insurance and health insurance premium. The higher the number, the higher the income. †Metropolitan included Seoul, Incheon and Gyeonggi-do Province. Urban included the rest of the cities, and rural included the rest of the provinces.

‡Type of institution was classified as number of beds. Clinic, hospital, general hospital and tertiary hospital each has $<30,30-100,100-500$ and over 500 beds, respectively.

§Cataract was defined as patients who had both diagnosis (H25-28, Q120) and surgery procedure (S5110-5111, S5117, S5119) code for cataract.

IGlaucoma was defined as patients who received prescription medications (Anatomical Therapeutic Chemical (ATC) code: S01E) for glaucoma.

anti-VEGF, antivascular endothelial growth factor; aSD, absolute standardised difference; CCI, Charlson Comorbidity Index; DM, diabetes mellitus; DME, diabetic macular oedema; nAMD, neovascular age-related macular degeneration. 
Table 2 Healthcare resource utilisation and direct medical costs for patients with DME treated with anti-VEGF, patients with DM without retinopathy and patients with nAMD treated with anti-VEGF during the 1-year follow-up period

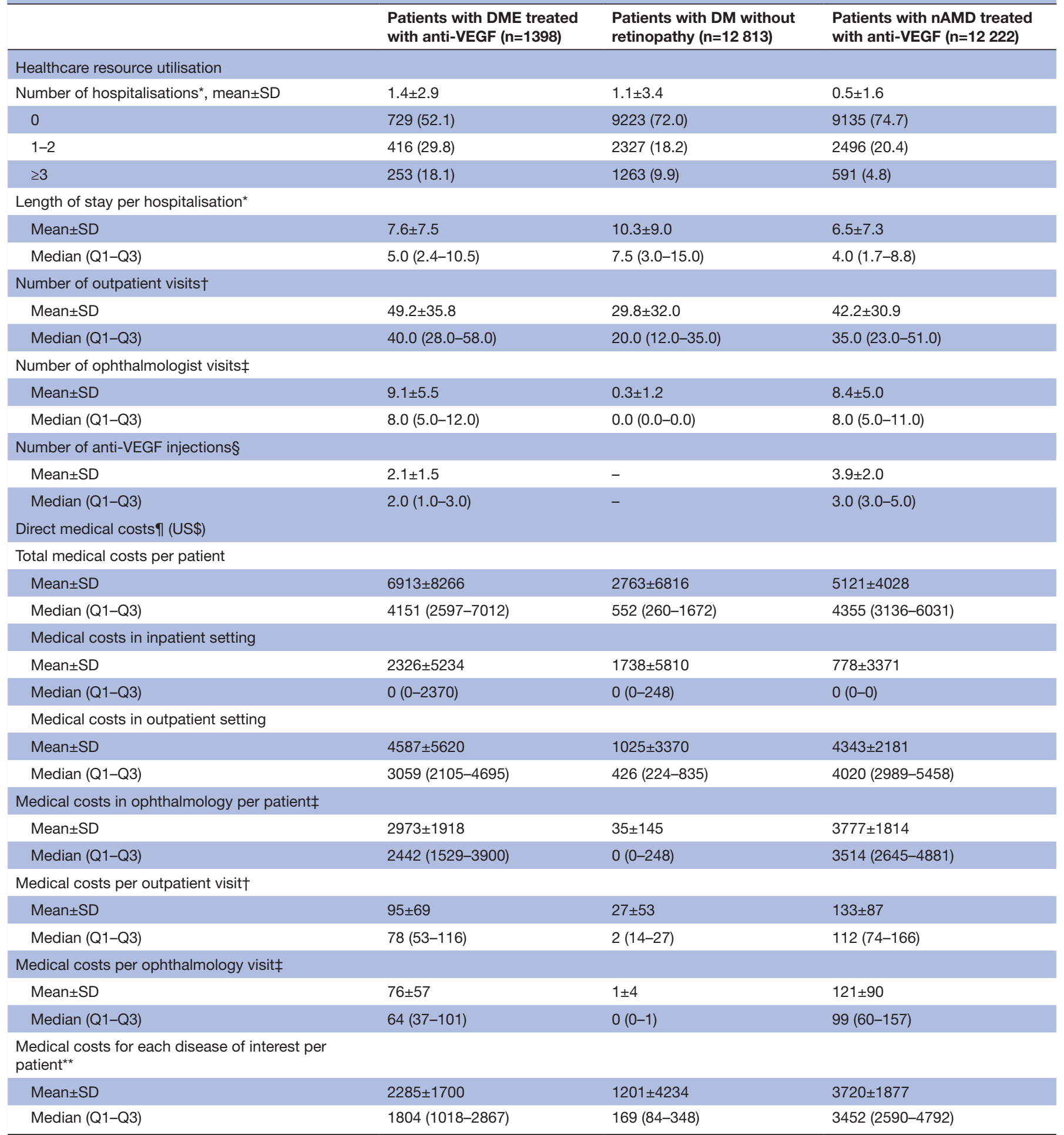

*Hospitalisations were identified by claims issued from inpatient visits through medical institute, public health institute and neuropsychiatric department. In the categories $(0,1-2, \geq 3)$ the number refers to the number of patients and percentage in parentheses.

†Outpatient visits were identified by claims issued from outpatient visits through medical institute, public health institute, neuropsychiatric department including day ward and haemodialysis.

fOphthalmologist visits were identified by claims with fundus examination issued from ophthalmology department.

SCalculated with the licensed anti-VEGFs (ranibizumab and aflibercept).

IDirect medical costs were converted from Korean won to US dollars based on the average exchange rate in 2015 (US $\$ 1=1179.1$ Korean won).

**The total medical costs for each disease of interest were defined as follows: DME, costs from all prescriptions for patients with DME (H360) and treated with licensed anti-VEGF, steroid or laser therapy; DM, costs from all prescriptions for patients with DM (E10-E14); nAMD, costs from all claims with a registration code for nAMD (V201).

anti-VEGF, antivascular endothelial growth factor; DM, diabetes mellitus; DME, diabetic macular oedema; nAMD, neovascular age-related macular degeneration; Q1, first quartile; Q3, third quartile. 
Table 3 OR for healthcare resource utilisation and cost ratios for direct medical costs of patients with DME treated with antiVEGF, relative to patients with DM without retinopathy

\begin{tabular}{|c|c|c|c|}
\hline & \multicolumn{3}{|l|}{ OR/cost ratio $(95 \% \mathrm{Cl})$} \\
\hline & Crude & Model 1* & Model 2† \\
\hline \multicolumn{4}{|l|}{ Healthcare resource utilisation } \\
\hline Number of outpatient visits§ & 1.25 (1.22 to 1.27$)$ & 1.24 (1.21 to 1.26$)$ & 1.27 (1.24 to 1.29$)$ \\
\hline Number of ophthalmologist visits & $12.29(11.38$ to 13.27$)$ & $12.33(11.42$ to 13.32$)$ & $12.35(11.44$ to 13.34$)$ \\
\hline Medical costs in inpatient setting & $1.00(0.76$ to 1.31$)$ & $1.03(0.78$ to 1.38$)$ & 1.05 (0.79 to 1.40$)$ \\
\hline Medical costs in outpatient setting & 2.51 (2.21 to 2.85$)$ & 4.78 (4.33 to 5.27$)$ & 5.70 (5.23 to 6.22$)$ \\
\hline Medical costs in ophthalmology per patient & 25.26 (22.11 to 28.87$)$ & 29.66 (25.73 to 34.18$)$ & 30.32 (26.30 to 34.97$)$ \\
\hline Medical costs per outpatient visit§ & 3.39 (3.13 to 3.67$)$ & 3.79 (3.53 to 4.08 ) & 4.06 (3.81 to 4.32$)$ \\
\hline
\end{tabular}

${ }^{*}$ Adjusted for age, sex, income level, DM duration, $\mathrm{CCl}$ and all comorbidities.

†Adjusted for 1-year medical costs per patient, number of outpatient visits, number of hospitalisations and mean length of stay per hospitalisation in the prior year, in addition to all covariates in model 1.

$\ddagger$ Hospitalisations were identified by claims issued from inpatient visits through medical institute, public health institute and neuropsychiatric department.

§Outpatient visits were identified by claims issued from outpatient visits through medical institute, public health institute, neuropsychiatric department including day ward and haemodialysis.

ๆOphthalmologist visits were identified by claims with fundus examination issued from ophthalmology department.

**1-year total medical costs per patient: the total medical costs per patient excluding the costs relevant to each disease.

anti-VEGF, antivascular endothelial growth factor; CCI, Charlson Comorbidity Index; DM, diabetes mellitus; DME, diabetic macular oedema.

DME was associated with 2.17 (95\% CI 1.83 to 2.58$)$ and 1.27 (95\% CI 1.24 to 1.29$)$ times more hospitalisations and outpatient visits than DM without retinopathy in model 2 (table 3). One-year medical costs of patients with DME were 4.66 times higher than those of patients with DM without retinopathy (95\% CI 4.17 to 5.20 ). Compared with the nAMD group, the number of hospitalisations in the DME group was $60 \%$ higher (OR 1.65; 95\% CI 1.39 to 1.85$)$ and the number of anti-VEGF injections was $50 \%$ lower (OR $0.50 ; 95 \%$ CI 0.46 to 0.54 ) in model 2 (table 4 ). The DME group had $16 \%$ lower 1-year medical costs than the nAMD group (95\% CI 0.78 to 0.90). Other medical costs were 29\%-33\% lower in general, but medical costs excluding the costs for each disease of interest for patients with DME were $66 \%$ higher than those for patients with nAMD (cost ratio $1.66 ; 95 \%$ CI 1.45 to 1.90 ).

\section{DISCUSSION}

Using real-world data, we examined the HRU and direct medical costs of patients with DME treated with antiVEGF and compared them with those of patients with DM without retinopathy and patients with nAMD treated with anti-VEGF. The mean number of hospital admissions and outpatient visits in the DME group was 1.4 and 49.2, and the mean number of anti-VEGF injections was 2.1. The mean medical costs were US\$6913 in the DME group. In the multivariate analysis with GLM after matching, patients with DME were likely to have had 1.3 times more outpatient visits and to have been hospitalised 2.2 times more often than patients with DM without retinopathy. One-year direct medical costs were 4.7 times higher in patients with DME. Compared with nAMD, DME was associated with 1.6 times more hospitalisations and $50 \%$ fewer licensed anti-VEGF injections.

Regarding the comparison between the DME group and the DM without retinopathy group, our results were consistent with those of previous studies. ${ }^{28}{ }^{29}$ Shea et $a l^{2}$ showed that the 1-year cost in the DME group was $31 \%$ higher than in the DM without retinopathy group. ${ }^{2}$ In addition, approximately three times as many patients with DME visited an ophthalmologist during a 1-year period after diagnosis compared with controls. The authors concluded that DME was a significant independent predictor of 1-year and 3-year total medical costs. In another retrospective cohort study, working-age patients with DME exhibited a significantly higher mean number of total healthcare visit days than patients with DM without DME (28.6 vs 16.9 days; $\mathrm{p}<0.001) .{ }^{29}$ Even when compared with DM with diabetic retinopathy without DME, DME was associated with higher rates of HRU and medical costs. $^{9} 10$

The overall HRU of patients with nAMD was lower in general than that of patients with DME, with the exception of an approximately twofold higher number of licensed anti-VEGF injections (3.9 vs 2.1). After adjusting the various factors in GLM, the OR regarding the anti-VEGF injections was significantly lower in the DME group. When restricting patients to first-ever users, the difference in the number of anti-VEGF injections remained (nAMD vs DME: 4.1 vs 2.1). 
Table 4 OR for healthcare resource utilisation and cost ratios for direct medical costs of patients with DME treated with antiVEGF, relative to patients with nAMD treated with anti-VEGF

\begin{tabular}{|c|c|c|c|}
\hline & \multicolumn{3}{|c|}{ OR/cost ratio $(95 \% \mathrm{Cl})$} \\
\hline & Crude & Model 1* & Model $2 \dagger$ \\
\hline \multicolumn{4}{|l|}{ Healthcare resource utilisation } \\
\hline Number of outpatient visits§ & 1.01 (1.00 to 1.03$)$ & $1.02(1.00$ to 1.04$)$ & 1.00 (0.99 to 1.02$)$ \\
\hline Number of ophthalmologist visits & 0.97 (0.93 to 1.01$)$ & 0.97 (0.93 to 1.01$)$ & 0.97 (0.93 to 1.01$)$ \\
\hline 1-year medical costs per patient & $0.88(0.81$ to 0.96$)$ & 0.85 (0.79 to 0.92$)$ & $0.84(0.78$ to 0.90$)$ \\
\hline Medical costs in inpatient setting & 1.09 (0.83 to 1.43$)$ & $1.06(0.80$ to 1.40$)$ & $1.06(0.80$ to 1.40$)$ \\
\hline Medical costs in outpatient setting & 0.73 (0.68 to 0.78$)$ & $0.72(0.67$ to 0.77$)$ & 0.71 (0.67 to 0.76$)$ \\
\hline Medical costs in ophthalmology visits per patient & $0.70(0.65$ to 0.75$)$ & $0.69(0.65$ to 0.75$)$ & $0.70(0.65$ to 0.75$)$ \\
\hline
\end{tabular}

*Adjusted for age, sex, income level, DM duration, $\mathrm{CCl}$ and all comorbidities.

$\dagger$ Adjusted for 1-year medical costs per patient, number of outpatient visits, number of hospitalisations and mean length of stay per hospitalisation in the prior year, in addition to all covariates in model 1.

¥Hospitalisations were identified by claims issued from inpatient visits through medical institute, public health institute and neuropsychiatric department.

§Outpatient visits were identified by claims issued from outpatient visits through medical institute, public health institute, neuropsychiatric department including day ward and haemodialysis.

१Ophthalmologist visits were identified by claims with fundus examination issued from ophthalmology department.

${ }^{* *}$ Calculated with the licensed anti-VEGFs (ranibizumab and aflibercept).

††1-year total medical costs per patient: the total medical costs per patient excluding the costs relevant to each disease.

anti-VEGF, antivascular endothelial growth factor; CCl, Charlson Comorbidity Index; DM, diabetes mellitus; DME, diabetic macular oedema; nAMD, neovascular age-related macular degeneration.

There are a few studies examining treatment patterns with anti-VEGF injections among patients with age-related macular degeneration (AMD) or DME in routine clinical practice. A study investigating the number of anti-VEGF injections administered with PRN treatment regimens for AMD and DME in a German hospital found that the median number of injections during the first year was 6 in patients with AMD and those with DME and the distribution of patients by number of injections was similar. ${ }^{30}$ In a study using the Danish National Patient Registry, the mean number of anti-VEGF injections in the first half of 2014 was 5.71 in patients with nAMD and 5.93 in patients with DME. ${ }^{18}$ When analysing US Medicare standard analytic claim files (2006-2010), patients with AMD received an average of 4.3 injections in the first year. ${ }^{19}$ Another study using the same database (2008-2010) showed that the mean number of anti-VEGF injection claims for DME per patient was 4.2. ${ }^{20}$ Studies conducted in Turkey reported the annual mean number of injections to be $4.1 \pm 1.9$ in patients with nAMD ${ }^{21}$ and 3.1-4.6 in patients with DME. ${ }^{22}$ The literature review indicates that the mean number of injections administered in a year in DME and nAMD should be comparable, although the number of loading doses of aflibercept for each disease is different in year 1 (3 in nAMD vs 5 in DME). However, our results showed that the mean number of injections in patients with nAMD was twofold higher than in patients with DME, indicating potential lack of treatment supported by the NHI among patients with DME.
This particular pattern was also shown in another Korean study.Jee $e t a l^{31}$ found that the mean (SD) number of licensed anti-VEGF injections in patients with nAMD was 4.87 (3.37), while that in patients with DME was 3.1 (1.98). Considering that the number of loading dose of aflibercept in the first year of treatment is higher in DME than nAMD (5 times vs 3 times), the very low number of anti-VEGF injection in DME amplifies the possibility of lack of treatment. The different copayment rate of patients with DME and nAMD may be one reason that explains this difference in the number of injections. In South Korea, nAMD has been designated as a rare and intractable disease since 2009, and patients with this disease receive benefit from a copayment decreasing policy (ie, extra benefit in national health insurance) in HRU. Because the copayment rate for patients with DME, which falls in the standard benefit scheme, is three to six times higher than for patients with nAMD $(30 \%-60 \%$ vs $10 \%)$, it could be burdensome for patients with DME to receive antiVEGF treatment. In addition, the relatively low income level of patients with DME might contribute to the low number of anti-VEGF injection. While the proportion of medical aid and patients in the fifth quantile of income level (the highest level) in patients with nAMD were $5.7 \%$ and $40.5 \%$, those in patients with DME were $22.2 \%$ and $20.7 \%$, respectively. Despite the low economic status, patients with DME were also likely to have more complicated comorbidity and thus might not afford to receive sufficient therapy. We could not 
disregard the possibility of switching from the licensed antiVEGF to an off-label treatment, bevacizumab, which could not be captured in our database. For patients with DME, the price of bevacizumab per one injection is cheaper than that of ranibizumab or aflibercept even if reimbursed. However, it surely indicated that ophthalmological treatment supported by the NHI may be insufficient for patients with DME compared with patients with nAMD.

The mean 1-year total medical costs were higher in the DME group than in the nAMD group (US\$6913 vs US\$5121). A previous study in South Korea reported that 1-year medical costs were US\$2995 and US\$1834 in patients with DME treated with anti-VEGF and patients with nAMD treated with anti-VEGF, respectively. ${ }^{31}$ The costs of that study were considerably different from those in the present study owing to differences in details of patient definition, but the trend of higher medical costs for the DME group compared with the nAMD group was similar. However, the costs relevant to ophthalmological care (the total medical costs for ophthalmological care per patient, the total medical costs per ophthalmologist visit and the total medical costs for each disease of interest per patient) in the DME group were lower than those of the nAMD group. In the GLM analysis with the matched cohort, we identified that DME was associated with 1-year medical costs that were $16 \%$ lower but with costs for comorbid diseases that were 1.66 times higher compared with nAMD. This indicates that patients with nAMD spent substantial money on treatment for nAMD, including antiVEGF injections, whereas patients with DME spent more money on treatments for diseases other than DME due to complicated comorbidity.

We used the nationwide administrative claims database to identify patients with DME, patients with DM without retinopathy and patients with nAMD. Use of administrative data is relatively free of the recall bias and non-response that may occur with the survey method. Furthermore, use of the administrative claims database allowed retrospective examination of the duration of DM in patients with DME and those with DM using 10-year data before the index date. Thus, claims data seem suitable to analyse the prevalence of disease and medical use and costs. In addition, to our knowledge, this is the first study to compare HRU and direct medical costs between a group of patients with DME treated with anti-VEGF and two comparison groups (patients with DM without retinopathy and patients with nAMD treated with anti-VEGF). To enhance comparability, we established a propensity score-matched cohort. In particular, the results from the comparison of the DME and nAMD groups show that healthcare use by patients can be affected by a pharmaceutical policy and may suggest the direction of future policy to decision makers. Moreover, GLM analyses were applied to the propensity score-matched cohort to identify the adjusted effect of DME relative to other disease statuses. These various statistical methods would contribute to reducing biases in estimating the effect of DME on HRU and medical costs.

This study had several limitations. First, because the clinical characteristics of patients with DME and those with nAMD are very different, propensity score matching does not completely control the confounders. Although we matched the groups by specific variables and adjusted for various confounders, residual confounding can still remain. Second, HRU and medical costs of patients with DME would have been underestimated. The NHI database does not include information on non-reimbursed drugs. Since information on the injection of bevacizumab, which has the same mechanism of action as ranibizumab and off-label treatment for DME and nAMD, is absent in the NHI database, the actual HRU and medical costs of patients would be higher than our results. However, it does not change the fact that patients with DME had higher HRU and medical costs overall and fewer licensed anti-VEGF injections. Third, there is a possibility of misclassification of patients with DME because there is no specific diagnosis code for DME in South Korea. Although we attempted to set the selection criteria reflecting clinical practice to distinguish patients with DME with accuracy, the misclassification may have remained and may have thus affected our results. Fourth, our findings may not be consistent if we look into the longer term, since we calculated the HRU and medical costs during a 1-year period. Intravitreal anti-VEGF treatment for DME versus nAMD can be different in the long term and thus the results should be interpreted with caution. Lastly, contrary to our interpretation, potential lack of anti-VEGF treatment in patients with DME might have contributed to their higher HRU and direct medical costs. Without timely treatment for DME, patients could have blurry vision, which might incur accidents such as falls, and subsequently result in ambulatory healthcare visit or hospital admission.

\section{CONCLUSION}

Compared with patients with DM without retinopathy, higher HRU and direct medical costs were incurred in patients with DME treated with anti-VEGF. The overall HRU of patients with DME was higher than that of patients with nAMD, while the use of licensed anti-VEGFs in patients with DME was half that in those with nAMD. The mean 1-year total medical costs of DME were higher than those of nAMD, but the mean medical costs for treatment of DME were lower than those for treatment of nAMD, which may reflect that patients with DME bear a heavy economic burden and that there is a potential lack of ophthalmological treatment supported by the NHI.

Contributors $\mathrm{H}-\mathrm{LJ}, \mathrm{HL}, \mathrm{DY}$ and $\mathrm{YL}$ designed the study. HL and DY played a key role in data analysis. H-LJ, HL, YL, JHK, DJ and J-YS participated in the interpretation of the results. YL administered the project. H-LJ and J-YS wrote the draft manuscript. All authors contributed to the final manuscript writing. J-YS is the guarantor of the study.

Funding This study was funded by Bayer Korea.

Competing interests J-YS reported receiving grants from the Ministry of Food and Drug Safety, the Ministry of Health and Welfare, the National Research Foundation of Korea, and Government-wide R\&D Fund for infectious disease research; and grants from pharmaceutical companies including Amgen, Pfizer, Hoffmann-La Roche, Dong-A ST and Yungjin, outside the submitted work. H-LJ, HL, DY and $\mathrm{J}$-YS reported grants from Bayer, the National Research Foundation of Korea, and Government-wide R\&D Fund for infectious disease research. YL is an employee of 
Bayer Korea. JHK is a consultant for Bayer Korea in this study. DJ has no conflicts of interest to declare.

\section{Patient consent for publication Not required.}

Ethics approval This retrospective study was approved by the Institutional Review Board (IRB) of Sungkyunkwan University in Korea (IRB no. SKKU-2018-04-018). The IRB official waiver of ethical approval was granted by the IRB of Sungkyunkwan University.

Provenance and peer review Not commissioned; externally peer reviewed.

Data availability statement Data may be obtained from a third party and are not publicly available. We used the National Health Insurance (NHI) database established by the National Health Insurance Service (NHIS) in South Korea. NHIS forbids transfer, rent or sale of the database to any other third party except the researchers who obtained approval for access to database (official website of NHIS https://nhiss.nhis.or.kr/bd/ab/bdaba000eng.do).

Supplemental material This content has been supplied by the author(s). It has not been vetted by BMJ Publishing Group Limited (BMJ) and may not have been peer-reviewed. Any opinions or recommendations discussed are solely those of the author(s) and are not endorsed by BMJ. BMJ disclaims all liability and responsibility arising from any reliance placed on the content. Where the content includes any translated material, BMJ does not warrant the accuracy and reliability of the translations (including but not limited to local regulations, clinical guidelines, terminology, drug names and drug dosages), and is not responsible for any error and/or omissions arising from translation and adaptation or otherwise.

Open access This is an open access article distributed in accordance with the Creative Commons Attribution Non Commercial (CC BY-NC 4.0) license, which permits others to distribute, remix, adapt, build upon this work non-commercially, and license their derivative works on different terms, provided the original work is properly cited, appropriate credit is given, any changes made indicated, and the use is non-commercial. See: http://creativecommons.org/licenses/by-nc/4.0/.

\section{ORCID iDs}

Ha-Lim Jeon http://orcid.org/0000-0002-9429-8711

Hyesung Lee http://orcid.org/0000-0001-6556-9984

Dongwon Yoon http://orcid.org/0000-0002-9369-0789

Jae Hui Kim http://orcid.org/0000-0001-8121-6353

Ju-Young Shin http://orcid.org/0000-0002-2791-1037

\section{REFERENCES}

1 Chen E, Looman M, Laouri M, et al. Burden of illness of diabetic macular edema: literature review. Curr Med Res Opin 2010;26:1587-97.

2 Shea AM, Curtis LH, Hammill BG, et al. Resource use and costs associated with diabetic macular edema in elderly persons. Arch Ophthalmol 2008;126:1748-54.

3 Hariprasad SM, Mieler WF, Grassi M, et al. Vision-related quality of life in patients with diabetic macular oedema. Br J Ophthalmol 2008:92:89-92.

4 Klein R, Klein BE, Moss SE, et al. The Wisconsin epidemiologic study of diabetic retinopathy. XV. The long-term incidence of macular edema. Ophthalmology 1995;102:7-16

5 Klein RKB, Moss SE. The epidemiology of ocular problems in diabetes mellitus.: Boston. MA: Blackwell Scientific Publications, 1991: 1-51.

6 Klein R, Knudtson MD, Lee KE, et al. The Wisconsin epidemiologic study of diabetic retinopathy XXIII: the twenty-five-year incidence of macular edema in persons with type 1 diabetes. Ophthalmology 2009;116:497-503.

7 Federation ID. Diabetes atlas. 8th ED. 8th edition ED, 2011.

8 Shaw JE, Sicree RA, Zimmet PZ. Global estimates of the prevalence of diabetes for 2010 and 2030. Diabetes Res Clin Pract 2010;87:4-14.

9 Lee LJ, Yu AP, Cahill KE, et al. Direct and indirect costs among employees with diabetic retinopathy in the United States. Curr Med Res Opin 2008;24:1549-59.

10 Happich M, Reitberger U, Breitscheidel L, et al. The economic burden of diabetic retinopathy in Germany in 2002. Graefes Arch Clin Exp Ophthalmol 2008;246:151-9.
11 Pershing S, Enns EA, Matesic B, et al. Cost-Effectiveness of treatment of diabetic macular edema. Ann Intern Med 2014;160:18-29.

12 Romero-Aroca P, Reyes-Torres J, Baget-Bernaldiz M, et al. st Century]]\&gt 2014;10:100-12.

13 Cho H, Choi KS, Lee JY, et al. Healthcare resource use and costs of diabetic macular oedema for patients with antivascular endothelial growth factor versus a dexamethasone intravitreal implant in Korea: a population-based study. BMJ Open 2019;9:e030930.

14 Park KH, Kim YY, Jo YJ, et al. Healthcare utilization and treatment patterns in diabetic macular edema in Korea: a retrospective chart review. J Korean Med Sci 2019;34:e118.

15 American Academy of Ophthalmology Retina/Vitreous Panel. Preferred practice Pattern $®$ Guidelines. age-related macular degeneration. San Francisco, CA: American Academy of Ophthalmology, 2015. www.aao.org/ppp

16 National Institute for Health and Care Excellence (NICE). Aflibercept for treating diabetic macular oedema. London (UK): National Institute for health and care excellence (NICE), 2015:46 P. (technology appraisal guidance; No. 346.

17 AOA Evidence-Based Optometry Guideline Development Group. Evidence-Based clinical practice guideline. eye care of the patient with diabetes mellitus. MO (US: American Optometric Association, 2014.

18 Vorum H, Olesen TK, Zinck J, et al. Real world evidence of use of anti-VEGF therapy in Denmark. Curr Med Res Opin 2016;32:1943-50.

19 Lad EM, Hammill BG, Qualls LG, et al. Anti-VEGF treatment patterns for neovascular age-related macular degeneration among Medicare beneficiaries. Am J Ophthalmol 2014;158:537-43.

20 Dugel PU, Layton A, Varma RB. Diabetic macular edema diagnosis and treatment in the real world: an analysis of Medicare claims data (2008 to 2010). Ophthalmic Surg Lasers Imaging Retina 2016;47:258-67.

21 Özkaya A, Karabaș L, Alagöz C, et al. Real-World outcomes of antiVEGF treatment for neovascular age-related macular degeneration in turkey: a multicenter retrospective study, Bosphorus retina Study Group report no: 1. Turk J Ophthalmol 2018;48:232-7.

22 Ozkaya A, Ozveren M, Demircan A. The real life data of ranibizumab use among the diabetic macular edema patients in turkey: documenting the improvement with clinical optimization during three consecutive years. Saudi J Ophthalmol 2018;32:175-9.

23 Lim S-S, Lee W, Kim Y-K, et al. The cumulative incidence and trends of rare diseases in South Korea: a nationwide study of the administrative data from the National health insurance service database from 2011-2015. Orphanet J Rare Dis 2019;14:49.

24 Park EC, Jang SI, Jeon SY, et al. Assessment of level of agreement in disease codes between health insurance claims data and medical records. Wonju: Health Insurance Review and Assessment service, 2018.

25 Internal Revenue Service. Yearly average currency exchange rates 2019. Available: https://www.irs.gov/individuals/internationaltaxpayers/yearly-average-currency-exchange-rates [Accessed 4 Dec, 2019].

26 Jayasekera J, Onukwugha E, Bikov K, et al. The economic burden of skeletal-related events among elderly men with metastatic prostate cancer. Pharmacoeconomics 2014;32:173-91.

27 Austin PC. An introduction to propensity score methods for reducing the effects of confounding in observational studies. Multivariate Behav Res 2011;46:399-424.

28 Wallick CJ, Hansen RN, Campbell J, et al. Comorbidity and health care resource use among commercially insured Non-Elderly patients with diabetic macular edema. Ophthalmic Surg Lasers Imaging Retina 2015;46:744-51.

29 Kiss S, Chandwani HS, Cole AL, et al. Comorbidity and health care visit burden in working-age commercially insured patients with diabetic macular edema. Clin Ophthalmol 2016;10:2443-53.

30 Wecker T, Ehlken C, Bühler A, et al. Five-Year visual acuity outcomes and injection patterns in patients with pro-re-nata treatments for AMD, DME, RVO and myopic CNV. Br J Ophthalmol 2017;101:353-9.

31 Jee D, Shin S, Suh J, et al. Economic evaluation of intravitreal injection of anti-vascular endothelial growth factor in ocular diseases: national evidence-based healthcare collaborating agency (NECA), 2017. 